\title{
Species differences in the distribution of infracuticular F-actin in outer hair cells of the cochlea
}

\author{
L. Carlisle ${ }^{1}$, G. Zajic ${ }^{2}$, R.A. Altschuler ${ }^{2}$, J. Schacht ${ }^{2}$ and P.R. Thorne ${ }^{3}$ \\ ${ }^{\prime}$ Electron Microscopy Unit, Institute of Laryngology and Otology, Royal National Throat, Nose and Ear Hospital, London, U.K., \\ ${ }^{2}$ Kresge Hearing Research Institute, The University of Michigan, Ann Arbor, Michigan, U.S.A. \\ and ${ }^{3}$ Department of Pathology, University of Auckland School of Medicine, Auckland, New Zealand
}

(Received 26 October 1987; accepted 17 January 1988)

\begin{abstract}
The distribution of filamentous actin (F-actin) in outer hair cells has been examined in several mammalian species using tetramethylrhodamine phalloidin, a specific marker for F-actin. The stereocilia and cuticular plates of the OHC in all species examined (pigmented guinea pig, hooded rat, chinchilla and squirrel monkey) contained F-actin; however, an infracuticular network of F-actin was present in OHC of the apical turns of the guinea pig cochlea but could not be identified in any other species examined.

F-actin; Outer hair cell; Phalloidin; Motility
\end{abstract}

\section{Introduction}

The cytoskeletal protein actin has been described in both sensory and supporting cells of the organ of Corti. Actin has been identified in the stereocilia and cuticular plates of both inner and outer hair cells, and in the pillar cells and Deiters cells, using anti-actin antibodies (Zenner, 1980; Flock et al., 1981, 1982; Slepecky and Chamberlain, 1985) and recently with phalloidin, a specific marker for the polymerized or filamentous form of actin (F-actin) (Zenner, 1986; Carlisle et al., 1987; Thorne et al., 1987).

In outer hair cells $(\mathrm{OHC})$ of the guinea pig, a spiralling network of F-actin descends from beneath the cuticle-free region of the apical pole of the cell towards the nucleus. This infracuticular network (ICN) of filaments in guinea pig OHC was originally described by Held (1926) and later shown to consist of actin using anti-actin antibody labelling (Zenner, 1980; Flock et al., 1982) and

Correspondence to: L. Carlisle, Electron Microscopy Unit, Institute of Laryngology and Otology, Royal National Throat, Nose and Ear Hospital, 330-332 Gray's Inn Road, London WCIX 8DX, U.K. rhodamine-labelled phalloidin (Zenner, 1986; Carlisle et al., 1987; Thorne et al., 1987).

In previous studies using rhodamine-labelled phalloidin we described a differential distribution of the ICN among $\mathrm{OHC}$ of the guinea pig organ of Corti (Carlisle et al., 1987; Thorne et al., 1987). The ICN was not found in all $\mathrm{OHC}$ of the guinea pig, but cells containing the ICN were in a consistent and distinctive pattern along the organ of Corti. It was present in $\mathrm{OHC}$ in the upper cochlear turns and most prominently in the third row $\mathrm{OHC}$ but was never present in $\mathrm{OHC}$ in the first cochlear turn. The purpose of the present study was to investigate the presence and distribution of the ICN in other mammalian species.

\section{Methods}

Hair cells from the organ of Corti of pigmented guinea pigs $(N=10)$, chinchillas $(N=10)$, hooded rats $(N=10)$ and squirrel monkeys $(N=4)$ were studied using surface preparations and cryosections and in vitro preparations of isolated $\mathrm{OHC}$. The methods used have been described in detail (Thorne et al., 1987), and are summarized as follows. 
Cochleae for surface preparations and cryosections were fixed by perilymphatic perfusion $(4.0 \%$ paraformaldehyde $/ 0.1 \%$ glutaraldehyde in $0.1 \mathrm{M}$ phosphate buffer, $230 \mathrm{mOsm}, \mathrm{pH}$ 7.4), then immersed in fixative for $1 \mathrm{~h}$. They were rinsed in buffer and the bony shell of the cochlea was removed. For surface preparations, the entire organ of Corti, still attached to the modiolus, was immersed in $330 \mathrm{~nm}$ phalloidin in phosphatebuffered saline (PBS) and placed on a rotor for 24 $h$ in the dark, then rinsed in buffer. The organ of Corti was then removed in segments and mounted on glass slides in 3:1 PBS/glycerol. For the cryosections, the entire organ of Corti and modiolus was decalcified in $2 \%$ ethylene diamine tetra-acetic acid (EDTA) and stored under refrigeration for $48 \mathrm{~h}$ with frequent changes of EDTA. After decalcification, sections were cut along the long axis of the cochlea and mounted on glass slides. These sections were then incubated in phalloidin (as above), placed in the dark for $24 \mathrm{~h}$, and rinsed in buffer. Living OHC were isolated from segments of the organ of Corti which had been incubated for $10 \mathrm{~min}$ in collagenase (1 $\mathrm{mg} / \mathrm{ml})$. The isolated cells were bathed in $33 \mathrm{~nm}$ phalloidin in Hanks' balanced salt solution for 20 min and rinsed in buffer. All specimens were studied by fluorescence microscopy.

\section{Results}

Phalloidin labelling was observed in the stereocilia and cuticular plate, and in a ring around the circumference of the cell at the apical pole, in all inner and outer hair cells of the species examined. The pillar cells, Deiters cells and junctional complexes in the reticular lamina contained F-actin as evidenced by intense phalloidin labelling.

Panel A of Fig. 1 shows a surface preparation view of the fourth turn of the organ of Corti of a guinea pig. In the first row $\mathrm{OHC}$, the cuticular plate is labelled by phalloidin with the exception of the cuticle-free area at its lateral margin. A ring of phalloidin label can be seen around the circumference of the cells in both the first and second row OHCs. In the third row, a cluster of phalloidin-labelled 'dots' are clearly visible beneath the cuticle-free region of the cuticular plate. 'Dots' of phalloidin are also present in the cuticle-free region in all of the second row $\mathrm{OHC}$ and a few of the first row $\mathrm{OHC}$, but they are much smaller than in the third row. Focussing through the surface preparation gave the impression that these 'dots' connected to form a structure which descended towards the nucleus of the cell. This impression was confirmed in radial cryosections of the fourth turn of guinea pig organ of Corti, as shown in panel B. A brightly stained structure, the ICN, is present inside the second and third row OHC. Using brightfield illumination we verified that the structure was actually inside the cell and although it descended for variable depths from cell to cell the ICN always disappeared above the nucleus.

A surface preparation of third turn organ of Corti from a chinchilla is shown in panel $C$. The cuticular plates and the circumference of the OHCs are labelled with phalloidin; however, there is no evidence of an ICN in the cuticle-free area at the lateral margin of the cells. The ICN was never seen in any $\mathrm{OHC}$ along the entire length of the chinchilla cochlea, nor was it observed in surface preparations of organ of Corti from the hooded rat or the squirrel monkey. The absence of an ICN was verified in radial cryosections of organ of Corti from chinchilla and hooded rat, as shown in panel D. Although the cuticular plates, Deiters cells and pillar cells are intensely labelled, the bodies of the OHCs are completely devoid of phalloidin labelling. The ICN was also not observed in living isolated $\mathrm{OHC}$ from chinchillas or from hooded rats.

In surface preparations of squirrel monkey organ of Corti, the phalloidin labelling in the cuticular plates of some OHCs showed a scalloped pattern, as illustrated in panel E. The actin substructure of the cuticular plate appeared to be anchored to the apical perimeter of these cells at discrete points. Cells in which this scalloped pattern was observed were most frequently found in the first row of OHCs, but there was no base to apex difference in their distribution.

\section{Discussion}

The distinctive infracuticular network of F-actin seen in some OHC of the guinea pig is not present within $\mathrm{OHC}$ of all mammalian species. However, apart from the ICN, F-actin in hair cells and supporting cells was found in the same locations 

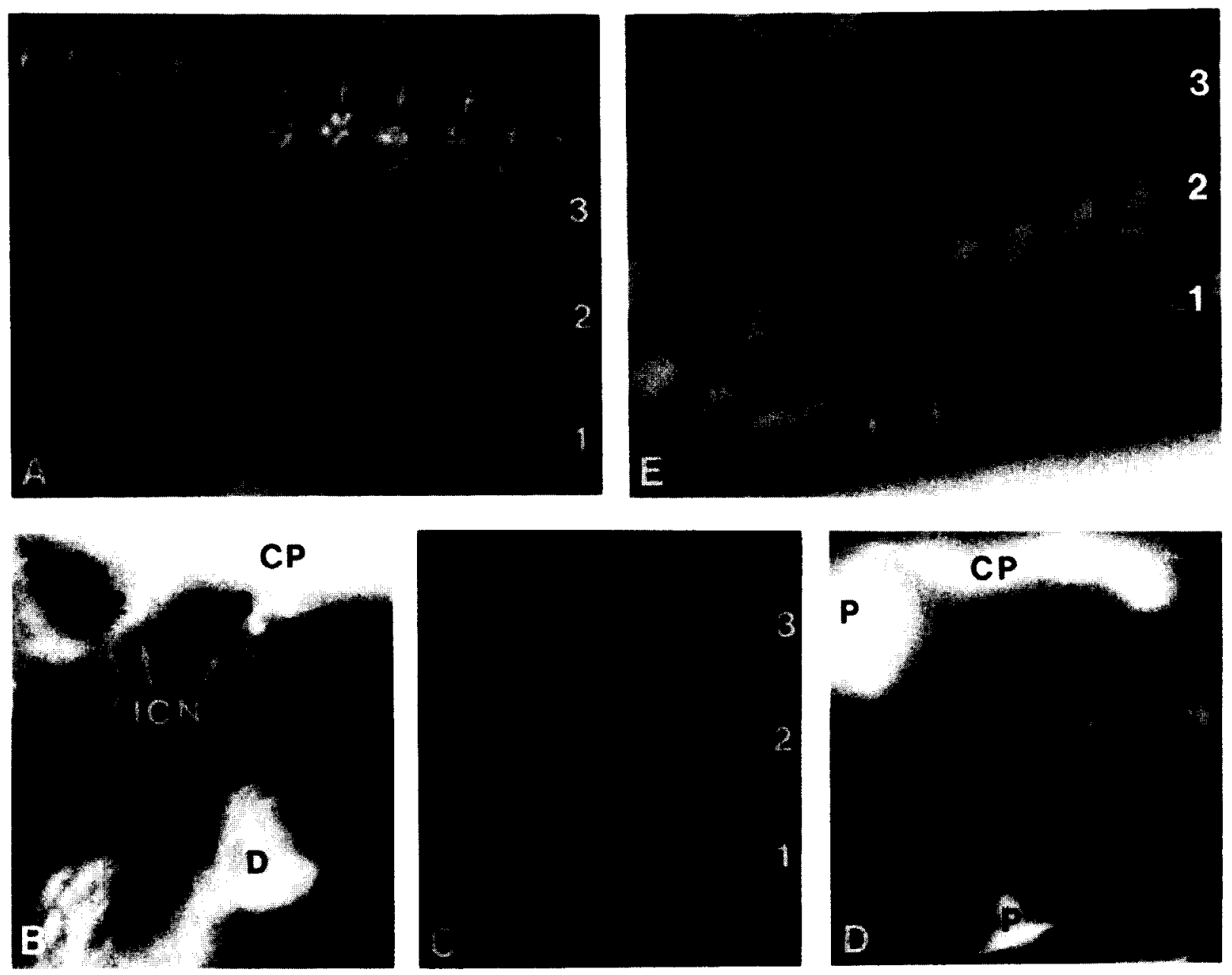

Fig. 1. Panel $\mathrm{A}$ is a surface preparation view from fourth turn guinea pig organ of Corti. The first row $\mathrm{OHC}$ are focussed at the level of the cuticular plates, which are phalloidin labelled. The second row OHC, at a slightly deeper level of focus, illustrates the ring of phalloidin staining observed around the circumference of the cells. The third row OHC, at yet a deeper level of focus, show the ICN, which appear as a cluster of brightly labelled dots (arrows) beneath the cuticle-free region of the cuticular plate. The ICN can also be seen in second and first row OHC although they are much smaller and less intensely labelled (open arrows). Panel B shows the ICN in second and third row $\mathrm{OHC}$ in a radial cryosection taken through fourth turn guinea pig organ of Corti. In the second row OHC, the ICN is tightly coiled but in the third row OHC it is more diffuse. Both forms were commonly observed. The cuticular plates (CP) and Deiters cells (D) are well labelled. Panel $\mathrm{C}$ shows a surface preparation from the third turn of the organ of Corti of a chinchilla at a similar level of focus as in panel A. Note the absence of any structure in the cuticle-free region of the cuticular plate in OHC 3 as compared with panel A. Panel D shows a radial cryosection from the upper turn of the rat cochlea, illustrating the absence of any structure comparable to the ICN illustrated in panel B. In the squirrel monkey, as shown in panel E, the phalloidin labelling suggested a scalloped shape for the actin substructure of the cuticular plate.

in all species. Previously, we have demonstrated that in the guinea pig, the ICN is differentially distributed in $\mathrm{OHC}$ along the organ of Corti, and this distribution has recently been confirmed (Flock, pers. comm.). The lack of ICN in rat $\mathrm{OHC}$ has also been confirmed and the ICN could not be identified in rabbit OHC (Flock, pers. comm.) or in $\mathrm{OHC}$ of the developing or mature mouse (Richardson, pers. comm.).

The scalloped appearance of the rhodamine labelling in the cuticular plate implies that the actin substructure is anchored to the lateral margin 
of the $\mathrm{OHC}$ at discrete points. We first observed this pattern in the cuticular plates of guinea pig OHCs, particularly in the OHCs of the first row, and a similar pattern was seen in this study for the squirrel monkey. Recently Steyger et al. (1987) reported a pattern of tubulin staining in the cuticular plates of guinea pig $\mathrm{OHC}$ which is the inverse of the actin pattern. This arrangement of actin and tubulin could be of structural importance in cuticular plate motility (Zenner, 1986).

We did not see the scalloped pattern in the cuticular plates of $\mathrm{OHC}$ of rat or chinchilla, but this may be related to our protocol. In the guinea pig, the scalloping was most readily observed in our early experiments where short incubation times were used and consequently the overall level of fluorescence was lower than in the present study. To enhance visualization of the ICN we increased incubation time and the intensity of the fluorescence was increased. Under these conditions the scalloping of the cuticular plate was less obvious. Since our primary interest in this study was the presence and distribution of the ICN, we used an incubation protocol designed to give an intense label, and this may account for the absence of scalloping observed in the chinchilla and rat.

Some aspects of the ultrastructure of the ICN have been described using transmission electron microscopy (Thorne et al., 1987). It consists of filaments which are longitudinally oriented in the cell and are associated with microtubules and mitochondria. In OHC from the lower turns of the guinea pig, where the ICN is not present, a collection of microtubules and mitochondria are found in the same intracellular location but without the filaments. Similarly, using differential interference contrast microscopy, a collection of organelles can be seen in the area beneath the cuticular plate in isolated hair cells of all species; however, this area only labels for F-actin in apical guinea pig OHC. Therefore the presence of a structure beneath the cuticular plate should not be interpreted as the ICN unless the appropriate labelling experiments have been performed.

A straightforward interpretation of the unique presence of the ICN in OHC of the apical portions of the guinea pig cochlea might be that an additional internal cytoskeleton is needed to provide structural support for these $\mathrm{OHC}$, which are considerably longer than those of other species in which measurements have been made. Living isolated outer hair cells of the apical cochlear turn in the guinea pig are $75 \mu \mathrm{m}$ long while apical $\mathrm{OHC}$ of the chinchilla are $60 \mu \mathrm{m}$ and apical OHC of the rat are only $31 \mu \mathrm{m}$ (Zajic and Schacht, 1987). The guinea pig is also unique in that its cochlea contains a fourth turn. Perhaps the combined effect of the geometry of the cochlea and the size of the OHC create the need for additional structural support for the mass of the organ of Corti.

Actin may be involved in hair cell motility in different ways. It has been suggested (Zenner, 1986; Flock et al., 1986) that polymerized cytoplasmic actin may be involved in the hair cell motility that has been demonstrated in vitro (Brownell et al., 1984; Zenner et al., 1985; Flock et al., 1986). Interaction of actin and myosin in the cuticular plate could lead to a change in cell length or tonicity by pulling on infracuticular and subplasmalemmal actin filaments (Zenner, 1986). Zenner (1986) has also reported an enhancement of phalloidin fluorescence in the ICN of isolated OHC when they were induced to contract by the application of ATP in the presence of calcium. It was suggested that this represents a rapid polymerization of actin filaments from a cytoplasmic pool of actin monomer (G-actin) during hair cell contraction. Our results could imply that in apical guinea pig $\mathrm{OHC}$ this pool of actin monomer is more readily stimulated to polymerization than in other species.

Any interpretation of the possible role of the ICN must remain speculative in the absence of definitive functional studies. If the ICN is involved in OHC motility, then the differences in distribution of the ICN in the guinea pig and its absence in other species demonstrated in this study may be an indication of differences in the mechanisms of hair cell motility among OHC of the guinea pig and between species. Whatever the role of the ICN in OHC function might be, since the majority of studies of isolated hair cells have been performed using cells from the apical half of the guinea pig cochlea, the finding that the ICN is unique to such cells suggests the possible necessity for some caution in interpreting the results obtained. 


\section{Acknowledgements}

This research was supported by NIH Program Project Grant NS 05785, NIH grant 11731, and by a USPHS International Research Fellowship No. TWO 3472 to P.R.T.

\section{References}

Brownell, W.E., Bader, C.R., Bertrand, D. and De Ribaupierre, Y. (1984) Evoked mechanical responses of isolated cochlear hair cells. Science 227, 194-196.

Carlisle, L., Thorne, P.R., Zajic, G., Schacht, J. and Altschuler, R.A. (1987) F-actin in the cochlea: Differential distribution between outer hair cells of different rows and turns. Abstr. Assoc. Res Otolaryngol. p. 202.

Flock, A., Cheung, H.C., Flock, B. and Utter, G. (1981) Three sets of actin filaments in sensory cells of the inner ear. J. Neurocytol. 10, 133-147.

Flock, A., Bretscher, A. and Weber, K. (1982) Immunohistochemical localization of several cytoskeletal proteins in inner ear sensory and supporting cells. Hear. Res. 6, 75-89.

Flock, A., Flock, B. and Ulfendahl, M. (1986) Mechanisms of movement of outer hair cells and a possible structural basis. Arch. Otorhinolaryngol. 243, 83-90.
Held, H. (1926) Die Cochlea der säuger und der vögel, ihre Entwicklung und ihr Bau. In: A. Bethe (Ed.), Handbuch der Normalen und Pathologischen Physiologie, Springer, Berlin, Vol. II, pp. 467-534.

Slepecky, N. and Chamberlain, S.C. (1985) Immunoelectron microscopic and immunofluorescent localization of cytoskeletal and muscle-like contractile proteins in inner ear sensory hair cells. Hear. Res. 20, 256-260.

Steyger, P.S., Furness, D.N., Hackney, C.M. and Richardson, G.P. (1987) The distribution of cytoskeletal proteins in the organ of Corti, demonstrated by immunocytochemistry. Abstr. Br. Soc. Audiol. p. 4.

Thorne, P.R., Carlisle, L., Zajic, G., Schacht, J. and Altschuler, R.A. (1987) Differences in the distribution of F-actin in outer hair cells along the organ of Corti. Hear. Res. 30, 253-266.

Zajic, G. and Schacht, J. (1987) Comparison of isolated outer hair cells from five mammalian species. Hear. Res. 26, 249-256.

Zenner, H.P. (1980) Cytoskeletal and muscle like elements in cochlear hair cells. Arch. Otorhinolaryngol. 230, 82-92.

Zenner, H.P. (1986) Motile responses in outer hair cells. Hear. Res. 22, 83-90.

Zenner, H.P., Zimmerman, U. and Schmitt, U. (1985) Reversible contractions of isolated mammalian cochlear hair cells. Hear. Res. 18, 127-133. 